\title{
Integration of Evora-InovGrid Smartmeters in a Consumer's SCADA System
}

\author{
R. Pereira, Joao Figueiredo, R. Melicio \\ CEM/IDMEC, University of Évora, Évora, \\ IDMEC, IST, University of Lisbon, Lisbon, \\ Portugal \\ jfig@uevora.pt \\ V.M.F. Mendes \\ University of Évora, Évora, \\ Lisbon Superior Engineering Institute, Lisbon \\ Portugal
}

\author{
J. Martins
Technology and Systems,
Nova de Lisboa, Lisbon \\ J. Martins
Center of Technology and Systems,
University Nova de Lisboa, Lisbon \\ J. Martins
Center of Technology and Systems,
University Nova de Lisboa, Lisbon \\ Portugal
}

Portugal

\author{
J.C. Quadrado \\ Lisbon Superior Engineering Institute, Lisbon \\ Portugal
}

\begin{abstract}
This paper develops an energy management system with smart meters integration for electricity consumers in a smart grid context. The integration of a distributor owned smart meter from the main Portuguese electricity distributor - EDP - is developed. The smart meter is connected to a common PC, that runs the Matlab Software and communicates to the SCADA system (Supervisory Control And Data Acquisition) using the OPC Protocol. The SCADA system supervises an industrial network of Programmable Logic Controllers (PLC). The developed control strategy implements a hierarchical cascade controller where inner loops are performed by local PLCs and the outer loop is managed by a centralized SCADA system, which interacts with the entire local PLC network.
\end{abstract}

Keywords-Smartmeters, Energy Management, SCADA Systems, Building Automation

\section{INTRODUCTION}

Smart grid promotes a bidirectional flow of electric power and communication between consumers and suppliers, throughout the inclusion of advanced Information and Communication Technologies (ICT) contributing for the passive end-consumers transformation into active players $[1,2]$.

Smart meters (SM) accurately measures both electricity consumption and production and communicates this data to the energy supplier. SM also allows to be integrated in home energy management systems, namely providing information about energy flow and price signals. Smart meters associated with their related infrastructures enable end-users to be included in the smart grid management context, as they provide information about electricity flow measurements and energy prices to end-consumers [3]. A SM system has control devices, sensors to identify parameters and devices to transfer data and command signals [4].

In this paper, emphasis is given to the smart technology which promotes the interface between the Portuguese power grid and the consumers of the InovGrid Project located in the city of Évora, Portugal. The main focus is to develop the distributor owned SM integration in a SCADA system supervising a PLC network in order to optimize the electricity consumption.

InovGrid is an innovator project based on a transformation process towards a new technical platform for power grid control and management. At technical level this project relies on 3rd generation technologies, in order to merge both communication network and power grid network. The InovGrid reference technical architecture is based on a hierarchical structure. The referred hierarchic structure has 3 levels: prosumer level, Medium-Low voltage (MV/LV) transformation level, data control and management level [5]. In prosumer level, energy boxes (EB) are implemented. The EB are SM that provide real consumption values as well as perform in-home energy management. In MV/LV transformation level, distribution transformer controllers (DTC) are implemented. DTCs allow load monitoring and power quality analysis. They manage the EBs, control the transformation station and control the public street lights. Finally, the data control and management level assembles the commercial information and performs the grid management.

This paper focuses on the prosumer level, as it is found to contribute clear and directly to consumers' economical savings, thus developing the consumers' awareness of the efficient use of electricity. The developed supervisory model integrates a SCADA system [6] connected to the Matlab Software [7] in order to implement advanced controllers. The communication channel selected for data transfer between SCADA and Matlab was the OPC protocol (Object Linking and Embedding -OLE- for Process Control) [8]. The SCADA system and PLC network integrates different types of information coming from the several technologies present in modern buildings such as Building Automation Systems (BAS). 